\title{
Seroprevalence of Encephalitozoon cuniculi and Toxoplasma gondii in domestic rabbits (Oryctolagus cuniculus) in China
}

\author{
Qing-Feng Meng ${ }^{1, \dagger}$, Wei-Lin Wang ${ }^{1, \dagger}$, Xiao-Ting Ni ${ }^{2, \dagger}$, Hai-Bin Li', Gui-Zhe Yao', Xiao-Lin Sun², Wei-Li Wang', \\ Wei Cong, \\ ${ }^{1}$ Jilin Entry-Exit Inspection and Quarantine Bureau, ChangChun, Jilin Province 130000, P. R. China; ${ }^{2}$ College of Veterinary Medicine, Gansu \\ Agricultural University, Lanzhou, Gansu Province 730030, P. R. China; ${ }^{3}$ College of Animal Science and Technology, Jilin Agricultural University, \\ Changchun, Jilin Province 130118, P. R. China
}

\begin{abstract}
The breeding of domestic rabbits (Oryctolagus cuniculus) for human consumption has a long tradition in China. Infections that can affect the production of meat or even be transmitted from animals to humans are important to monitor, especially for public health reasons as well as for their impact on animal health. Thus, a total of 1,132 domestic rabbit sera from 4 regions in China were collected for serological screening for Encephalitozoon cuniculi and for Toxoplasma gondii by ELISA and modified agglutination test (MAT), respectively. Antibodies to $E$. cuniculi were detected in 248/1,132 (21.9\%) sera tested while antibodies against $T$. gondii revealed a seroprevalence of 51/1,132 (4.5\%). We believe that the present results are of epidemiological implications and public health importance due to the acknowledged susceptibility of humans to $E$. cuniculi and $T$. gondii infections. Therefore, routine screening tests of domestic rabbits are proposed considering the zoonotic potential of these parasites.
\end{abstract}

Key words: Encephalitozoon cuniculi, Toxoplasma gondii, domestic rabbit, seroprevalence, China

Neurological diseases are usually detected in domestic rabbits, and the main clinical differential diagnoses include encephalitozoonosis, toxoplasmosis, peripheral vestibular disorders such as tympanitis and labyrinthitis, mite infestation, vitamin E deficiencies, and traumatic events [1].

Encephalitozoon cuniculi is a single-celled, spore-forming, obligate intracellular parasite in the phylum Microsporidia [2]. The microsporidian E. cuniculi principally affects rabbits but can also be found in humans. Human encephalitozoonosis is mostly found in immunocompromised patients, including HIV patients, organ transplant recipients, and cancer patients who are being treated with chemotherapy [3]. Humans get infected mainly through the ingestion of food or water contaminated with spores from urine of infected animals [4]. Rabbits suffering from encephalitozoonosis display various clinical symptoms from asymptomatic to sudden death. The disease

- Received 23 June 2015, revised 13 September 2015, accepted 14 September 2015.

*Corresponding author (messicw@163.com)

†These authors contributed equally to this work.

(c) 2015, Korean Society for Parasitology and Tropical Medicine

This is an Open Access article distributed under the terms of the Creative Commons Attribution Non-Commercial License (http://creativecommons.org/licenses/by-nc/3.0) which permits unrestricted non-commercial use, distribution, and reproduction in any medium, provided the original work is properly cited. may be acute or chronic, and the clinical symptoms are induced by lesions existing in the central nervous system, kidney, or eye $[2,3]$.

Toxoplasma gondii is a globally distributed pathogen infecting animals and humans [5]. Infections with $T$. gondii in rabbits may result in fatal toxoplasmosis [6,7]. Moreover, rabbits are infected by ingesting the forage or water contaminated with sporulated oocysts resulting in the formation of tissue cysts in muscles which is infectious for other intermediate or definitive hosts [8]. In fact, T. gondii type III strains have been isolated from domestic rabbits in China, suggesting that domestic rabbits could act as potential reservoirs for T. gondii transmission [9]. Actually, consumption of rabbit meat has been reported to be associated with T. gondii infection in humans in Durango, Mexico [10].

Domestic rabbits are consider to be a potential source of $T$. gondii infection in humans, so it is very important to know the risk factors of transmission through consuming meat. Although human encephalitozoonosis is reported mostly in immunocompromised individuals, the infection can be transmitted from animals to farmers by spores in urine and even diffuse via the environment. Moreover, evaluating how these par- 
asites influence animal health is of importance because domestic rabbits have an economic effect on agriculture. The breeding of domestic rabbit (Oryctolagus cuniculus) for human consumption has a long tradition in China. Rabbit industry is one of the green economy industries which is an emerging and promising animal husbandry with broad space for development, and its products have brought great wealth to the rural people in China. At present, China is the world's superpower of breeding rabbits, and domestic rabbits yield high quality meat and hair which are transported to many parts of home and abroad.

However, disease is the main challenge for the rabbit industry. Moreover, the public health concerns associated with E. Cuniculi and $T$. gondii definitely indicate that it is needed to carry out an epidemiological survey of these infections in animals, especially for those which can be used as a source of food. However, limited data is available on the prevalence of $E$. $\mathrm{Cu}$ niculi and T. gondii infection in rabbits in China. The present study aimed to report the seroprevalence of E. cuniculi and $T$. gondii in rabbit farms representing 4 provinces of China (Liaoning Province, Jilin Province, Heilongiiang Province, and Inner Mongolia Autonomous Region) where infection could have economical and public health implications.

The study was approved by the Ethics Committee of Jilin Entry-Exit Inspection and Quarantine Bureau, China. From
January 2013 to May 2014, a total of 1,132 blood samples were collected from 12 rabbit farms over 50 head registered in the census of the zone in Liaoning, Jilin, and Heilongiiang Province and Inner Mongolia Autonomous Region (Fig. 1). Rabbits from each farm were selected randomly using a table of random digits. Representative farm samples were selected according to the estimated number in each area. The small-scale farms (population less than 50) were not included because the animal owner was not present to give permission. The sample size (approximately $5 \%$ of rabbits) was determined in each farm in order to estimate results at farm-level. Moreover, all of the animals used in the present study were clinically healthy. From each rabbit, approximately $2 \mathrm{ml}$ of blood was collected from the marginal ear vein or post mortem. After centrifugation at 3,000 rpm for $10 \mathrm{~min}$, sera were collected and stored at $-20^{\circ} \mathrm{C}$ until use. Handling of rabbits was in compliance with the Animal Ethics Procedures and Guidelines of the People's Republic of China. Information about species, ages, geographic origin, and gender were acquired from the farm administrators.

A commercial ELISA kit (Medicago, Uppsala, Sweden) was used to detect and verify antibodies against $E$. cuniculi in rabbit sera [11]. The procedure was performed following the kit manufacturer's instructions. The domestic rabbit serum samples were tested for $T$. gondii antibodies by the modified agglutination test (MAT) as described previously [7]. Briefly, sera were

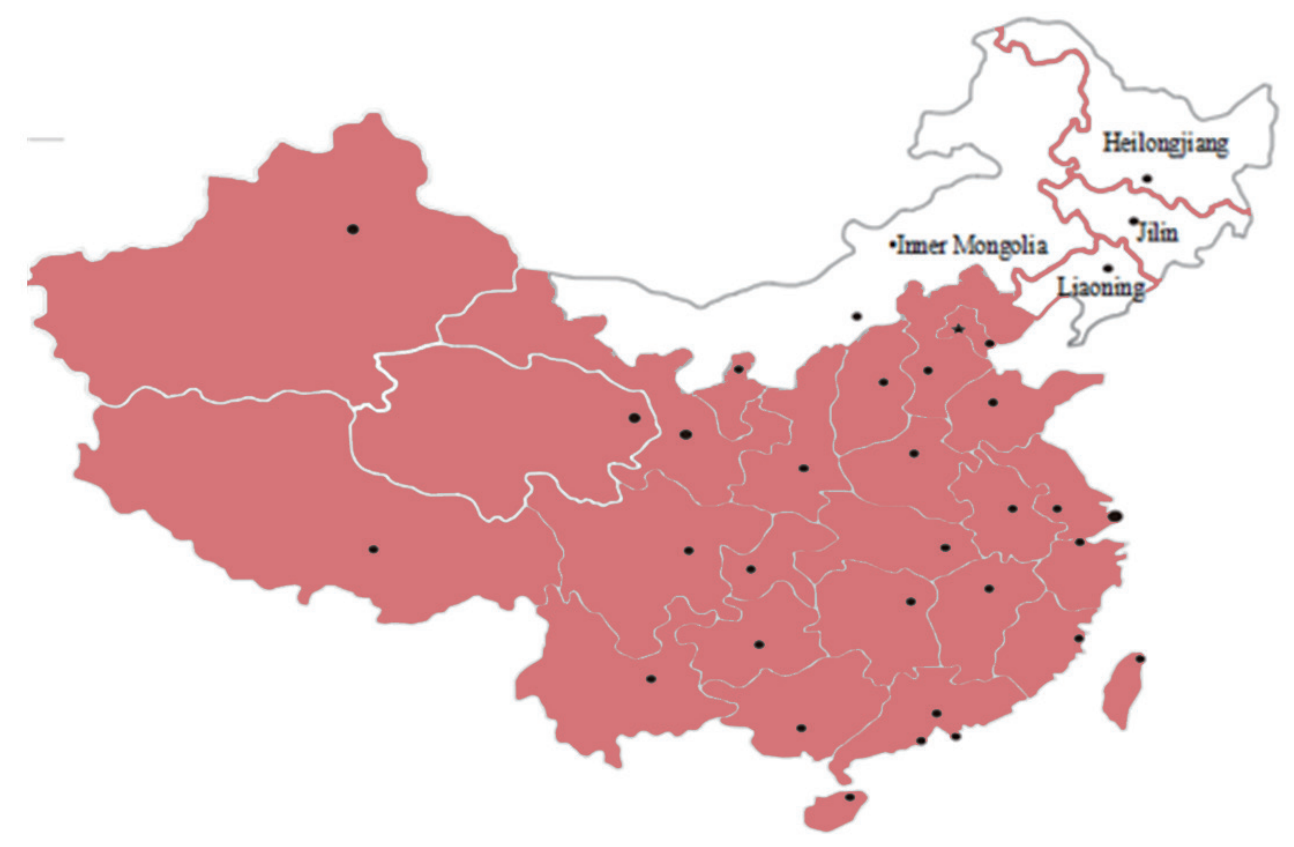

Fig. 1. Geographic distribution of sampling regions for rabbits in China. 
added to the "U" bottom of 96-well microtiter plates, and diluted 2-folds starting from 1:25 to 1:400. Domestic rabbits sera with MAT titers of 1:25 or higher were considered positive for T. gondii antibodies based on a previous study [7], those sera with doubtful reactions were retested, and positive and negative controls were included in each test.

Differences in the seroprevalence of E. cuniculi-infected and T. gondii-infected domestic meat rabbits among different variables including regions, age, gender, species, and sampling time were analyzed using a chi-square test by SAS (Statistical Analysis System, version 9.3), 95\% confidence intervals (CI) are given. The differences were considered statistically significant when $P<0.05$.

Antibodies against $E$. cuniculi were detected in 248 (21.9\%) of 1,132 serum samples by ELISA. Positive rabbits were found in all sampling regions, varying from $17.3 \%$ to $30.9 \%$, and rabbits in Heilongiiang Province (17.3\%) had a significantly lower seroprevalence than that in other regions $(P=0.001)$ (Table 1). In terms of sampling seasons, $E$. cuniculi seroprevalence varied in rabbits among 4 different seasons, ranging from $10.6 \%$ in winter to $29.0 \%$ in summer $(P<0.001)$. Among different species of meat rabbits, the highest seroprevalence was seen in New Zealand rabbits (23.7\%), followed by California rabbits (22.0\%), and Chinese rabbits (19.9\%). Adult rabbits (27.3\%) had a significantly higher seroprevalence than young rabbits $(17.4 \%)(P<0.001)$. E. cuniculi seroprevalence in male and female sika deer were $21.1 \%$ and $22.5 \%$, respectively.

Antibodies to T. gondii were found in $51(4.5 \%)$ of 1,132 domestic meat rabbits, with titers of 1:25 in 15, 1:50 in 18, 1:100 in $9,1: 200$ in 5 , and 1:400 in 4. Seropositive rabbits were found in all 4 sampling regions, ranging from $3.5 \%$ in Inner Mongolia Autonomous Region to 6.5\% in Liaoning Province (Table 1). Among different species of meat rabbits, the highest seroprevalence was seen in California rabbits (5.1\%), followed by New Zealand rabbits (4.3\%) and Chinese rabbits (4.2\%). In terms of sampling times, T. gondii seroprevalence in winter $(2.8 \%)$ was lower than in other seasons $(P=0.084)$. $T$. gondii seroprevalence in young rabbits and adult rabbits were $3.2 \%$ and $6.1 \%$, respectively. Male rabbits had a higher prevalence $(4.6 \%)$ compared to females (4.4\%) (Table 1$)$.

The serological results of this study revealed an overall seropositive rate $(21.9 \%)$ of $E$. cuniculi infection in domestic meat rabbits detected by ELISA, which was much lower than the $67.8 \%$ prevalence among 171 rabbits in Taiwan [11], 58.5\% in 118 rabbits in Austria [1], and 67.2\% in pet rabbits in Italy [12]. However, the prevalence was much higher than the values of 15\% in farmed domestic rabbits in Egypt [13], 7.7\% in New Zealand rabbits in Turkey [14], and 22.6\% in pet rabbits

Table 1. General characteristics of the 1132 domestic rabbits studied and seroprevalence of $E$. cuniculi and T. gondii infection

\begin{tabular}{|c|c|c|c|c|c|c|c|}
\hline \multirow{2}{*}{ Characteristics } & \multirow{2}{*}{$\begin{array}{l}\text { Rabbits tested } \\
\text { (No.) }\end{array}$} & \multicolumn{3}{|c|}{ Encephalitozoon cuniculi } & \multicolumn{3}{|c|}{ Toxoplasma gondii } \\
\hline & & No. positive & $\%(95 \%$ Cl) & $P$-value & No. positive & $\%(95 \%$ Cl) & $P$-value \\
\hline \multicolumn{8}{|l|}{ Age } \\
\hline Young & 620 & 108 & $17.4(14.4-20.4)$ & \multirow{2}{*}{$<0.001$} & 20 & $3.2(1.8-4.6)$ & \multirow[t]{2}{*}{0.022} \\
\hline Adult & 512 & 140 & $27.3(23.5-31.2)$ & & 31 & $6.1(4.0-8.1)$ & \\
\hline \multicolumn{8}{|l|}{ Gender } \\
\hline Male & 479 & 101 & $21.1(17.4-24.7)$ & \multirow{2}{*}{0.567} & 22 & $4.6(2.7-6.5)$ & \multirow{2}{*}{0.903} \\
\hline Female & 653 & 147 & $22.5(19.3-25.7)$ & & 29 & $4.4(2.9-6.0)$ & \\
\hline \multicolumn{8}{|l|}{ Species } \\
\hline Chinese Rabbit & 382 & 76 & 19.9 (15.9-23.9) & \multirow[t]{3}{*}{0.431} & 16 & $4.2(2.2-6.2)$ & \multirow[t]{3}{*}{0.816} \\
\hline California Rabbit & 354 & 78 & $22.0(17.7-26.4)$ & & 18 & $5.1(2.8-7.4)$ & \\
\hline New Zealand Rabbit & 396 & 94 & $23.7(19.6-27.9)$ & & 17 & $4.3(2.3-6.3)$ & \\
\hline \multicolumn{8}{|l|}{ Region } \\
\hline Liaoning Province & 246 & 76 & $30.9(25.1-36.7)$ & \multirow[t]{2}{*}{0.001} & 16 & $6.5(3.4-9.6)$ & \multirow[t]{2}{*}{0.337} \\
\hline Heilongjiang Province & 243 & 42 & $17.3(12.5-22.0)$ & & 9 & $3.7(1.3-6.1)$ & \\
\hline Inner Mongolia Autonomous Region & 289 & 64 & $22.2(17.4-26.9)$ & & 10 & $3.5(1.4-5.6)$ & \\
\hline \multicolumn{8}{|l|}{ Season } \\
\hline Spring & 283 & 67 & 23.7 (18.7-28.6) & \multirow[t]{4}{*}{$<0.001$} & 15 & $5.3(2.7-7.9)$ & \multirow[t]{4}{*}{0.084} \\
\hline Summer & 283 & 82 & $29.0(23.7-34.3)$ & & 19 & $6.7(3.8-9.6)$ & \\
\hline Autumn & 282 & 68 & $24.1(19.1-29.1)$ & & 9 & $3.2(1.1-5.2)$ & \\
\hline Winter & 284 & 30 & $10.6(7.0-14.1)$ & & 8 & $2.8(0.9-4.7)$ & \\
\hline Total & 1,132 & 248 & $21.9(19.5-24.3)$ & & 51 & $4.5(3.3-5.7)$ & \\
\hline
\end{tabular}


in Korea [15]. Such differences may be associated with several factors, such as the geographical and climatic conditions, the size and species of the rabbits tested, farm management, as well as the specificity and sensitivity of the detection methods.

Comparison of the infection rate of $E$. cuniculi by gender showed that 101/479 (21.1\%) in male and 147/653 (22.5\%) in female were seropositive. Furthermore, comparison of the infection rate by age showed that adult rabbits had a significantly higher positivity for $E$. cuniculi than young rabbits. This is in agreement with the study conducted on pet rabbits in Italy [12]. This phenomenon can be explained by the significant effect of age reported in the present study in consideration that maternal antibodies are passed on to the offspring and are present until 4 weeks of age [12,16]; from 4 to 8 weeks of age the animals are seronegative. In this study, most of the young rabbits tested were 1- and 2-months old, and would, thus, have fallen into this latter category.

The present results show a significantly lower E. cuniculi seropositivity for Heilongiiang Province than the other 3 regions in China $(P=0.001)$. This may be because the rabbit habitat in Heilongiiang Province has a lower population density. Moreover, this study shows significant differences in seropositivity rates among different seasons $(P<0.001)$. Rabbits in winter have the lowest risk of being infected compared to rabbits in other seasons. This may be caused by cold and dry climates in winter, which could reduce the infectivity of spores [17].

Demand for rabbit meat for human consumption is growing due to the leanness of their meat. Furthermore, there are no regulations concerning sale and slaughter of these animals in China. Therefore, infection in rabbits could be of importance in the epidemiology of toxoplasmosis. However, seroprevalence data from domestic rabbits are limited in China [9]. In our study, we detected $4.5 \% \mathrm{~T}$. gondii antibodies prevalence in domestic rabbits. Adult rabbits had a higher T. gondii seroprevalence (6.1\%) than young rabbits (3.2\%). This difference may be explained by that adult rabbits have more time to become infected with T. gondii. Moreover, this study revealed that the season was a significant risk factor associated with T. gondii seroprevalence. Rabbits in summer (6.7\%) and spring (5.3\%) had a higher seroprevalence than that in autumn (3.2\%) and winter $(2.8 \%)$. Such significant seasonal differences in prevalence may be due to different climates in different seasons. The warm and relatively humid climates in spring and summer are favorable for development of T. gondii oocysts, whereas the cold and dry climates in autumn and winter may be less favor-

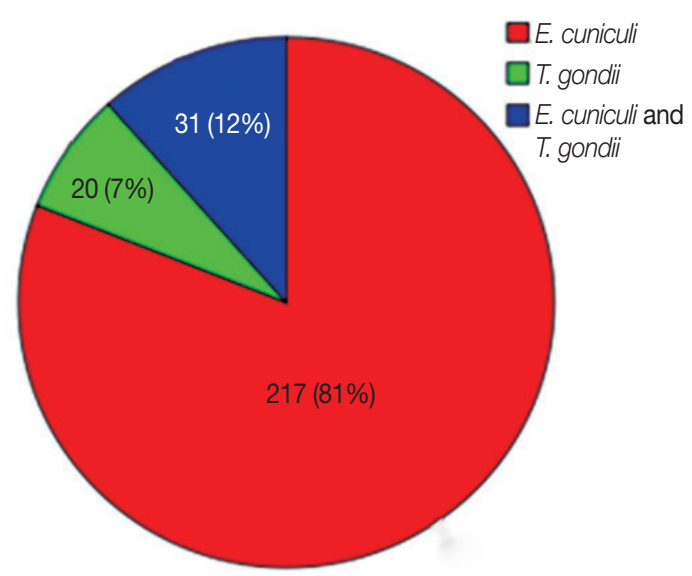

Fig. 2. Summary of distribution (number and percentage) of infected rabbits.

able for spreading of $T$. gondii oocysts.

The seroprevalence of E. cuniculi recorded in the present study was higher than that of $T$. gondii in agreement with previous studies $[4,18]$. Direct transmission of E. cuniculi by spores excreted in urines of infected animals would have apparently promoted the transmission of the infection in rabbit flocks [4]. Interestingly, E. cuniculi positive rabbits in the present study were significantly more often positive for anti-T. gondii antibodies than those animals of $E$. cuniculi negative group. Moreover, concomitant infection was common in the present study (Fig. 2). This co-occurrence might be attributable to a higher susceptibility after concomitant infection.

In conclusion, this study revealed that E. cuniculi is present and widespread among domestic rabbits. Information about $E$. cuniculi seroprevalence is important not only on account of the zoonotic potential of this parasite but also because the parasite can cause considerable economic losses due to mortality of host animals. Moreover, we could conclude that, albeit the lower rates of T. gondii infections in our domestic rabbits, the risk of parasite transmission caused by rabbit meat consumption is still considerable. Considering zoonotic potential and public health concerns, the authors strongly advise that serology tests should be included in routine health screening for domestic rabbits, especially for meat rabbits.

\section{ACKNOWLEDGMENT}

This work was supported by a grant from Quality Inspection Special Public Welfare Industry Research, China (no. 201410061). 


\section{CONFLICT OF INTEREST}

The authors declare that they have no competing interests.

\section{REFERENCES}

1. Gruber A, Pakozdy A, Weissenböck H, Csokai J, Künzel F. A retrospective study of neurological disease in 118 rabbits. J Comp Pathol 2009; 140: 31-37.

2. Didier ES. Microsporidiosis: an emerging and opportunistic infection in humans and animals. Acta Trop 2005; 94: 61-66.

3. Mathis A, Weber R, Deplazes P. Zoonotic potential of the microsporidia. Clin Microbiol Rev 2005; 18: 423-445.

4. Neumayerová H, Juránková J, Jeklová E, Kudláčková H, Faldyna M, Kovařčík K, Jánová E, Koudela B. Seroprevalence of Toxoplasma gondii and Encephalitozoon cuniculi in rabbits from different farming systems. Vet Parasitol 2014; 204: 184-190.

5. Dubey JP. Toxoplasmosis of Animals and Humans, 2nd ed. Boca Raton, Florida, USA. CRC Press. 2010, pp 1-313.

6. Dubey JP, Brown CA, Carpenter JL, Moore JJ. Fatal toxoplasmosis in domestic rabbits in the USA. Vet Parasitol 1992; 44: 305309.

7. Alvarado-Esquivel C, Alvarado-Esquivel D, Villena I, Dubey JP. Seroprevalence of Toxoplasma gondii infection in domestic rabbits in Durango State, Mexico. Prev Vet Med 2013; 111: 325-328.

8. Dubey JP. Toxoplasmosis-a waterborne zoonosis. Vet Parasitol 2004; 126: 57-72.

9. Zhou Y, Zhang H, Cao J, Gong H, Zhou J. 2013. Isolation and genotyping of Toxoplasma gondii from domestic rabbits in China to reveal the prevalence of type III strains. Vet Parasitol 2013;
193: 270-276.

10. Alvarado-Esquivel C, Torres-Berumen JL, Estrada-Martínez S, Liesenfeld O, Mercado-Suarez MF. Toxoplasma gondii infection and liver disease: a case-control study in a northern Mexican population. Parasit Vectors 2011; 4: 75.

11. Tee KY, Kao JP, Chiu HY, Chang MH, Wang JH, Tung KC, Cheng FP, Wu JT. Serological survey for antibodies to Encephalitozoon cuniculi in rabbits in Taiwan. Vet Parasitol 2011; 183: 68-71.

12. Dipineto L, Rinaldi L, Santaniello A, Sensale M, Cuomo A, Calabria M, Menna LF, Fioretti A. Serological survey for antibodies to Encephalitozoon cuniculi in pet rabbits in Italy. Zoonoses Public Health 2008; 55: 173-175.

13. Ashmawy KI, Abuakkada SS, Awad AM. Seroprevalence of antibodies to Encephalitozoon cuniculi and Toxoplasma gondii in farmed domestic rabbits in Egypt. Zoonoses Public Health 2011; 58: 357-364.

14. Ozkan O, Ozkan AT, Zafer K. Encephalitozoonosis in New Zealand rabbits and potential transmission risk. Vet Parasitol 2011; 179: 234-237.

15. Shin JC, Kim DG, Kim SH, Kim S, Song KH. Seroprevalence of Encephalitozoon cuniculi in pet rabbits in Korea. Korean J Parasitol 2014; 52: 321-323.

16. Lyngset A. A survey of serum antibodies to Encephalitozoon cuniculi in breeding rabbits and their young. Lab Anim Sci 1980; 30: 558-561.

17. Waller T. Sensitivity of Encephalitozoon cuniculi to various temperatures, disinfectants and drugs. Lab Anim 1979; 13: 227-230.

18. Jeklova E, Jekl V, Kovarcik K, Hauptman K, Koudela B, Neumayerova $\mathrm{H}$, Knotek Z, Faldyna M. Usefulness of detection of specific IgM and IgG antibodies for diagnosis of clinical encephalitozoonosis in pet rabbits. Vet Parasitol 2010; 170:143-148. 
